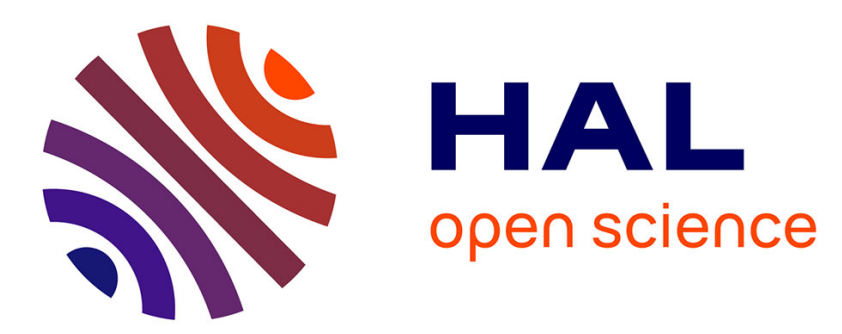

\title{
Effect of manipulation of the feet and ankles on postural control in elderly adults
}

\author{
Jacques Vaillant, Nicolas Vuillerme, Audrey Janvy, François Louis, Renaud \\ Braujou, Robert Juvin, Vincent Nougier
}

\section{- To cite this version:}

Jacques Vaillant, Nicolas Vuillerme, Audrey Janvy, François Louis, Renaud Braujou, et al.. Effect of manipulation of the feet and ankles on postural control in elderly adults. Brain Research Bulletin, 2008, 75 (1), pp.18-22. hal-00282239

\section{HAL Id: hal-00282239 \\ https://hal.science/hal-00282239}

Submitted on 26 May 2008

HAL is a multi-disciplinary open access archive for the deposit and dissemination of scientific research documents, whether they are published or not. The documents may come from teaching and research institutions in France or abroad, or from public or private research centers.
L'archive ouverte pluridisciplinaire HAL, est destinée au dépôt et à la diffusion de documents scientifiques de niveau recherche, publiés ou non, émanant des établissements d'enseignement et de recherche français ou étrangers, des laboratoires publics ou privés. 
3

4

5

6

7

8

9

10

11

\section{Effect of manipulation of the feet and ankles on postural control in elderly adults}

(1)
3.

Vaillant $\mathrm{J}^{12^{*}}$, Vuillerme $\mathrm{N}^{1}$, Janvy $\mathrm{A}^{2}$, Louis $\mathrm{F}^{2}$, Braujou $\mathrm{R}^{2}$, Juvin $\mathrm{R}^{3}$, Nougier $\mathrm{V}^{1}$. (1) (1)

(1)

8

${ }^{1}$ Laboratoire TIMC-IMAG, Equipe SPM, Université Joseph Fourier, UMR CNRS 5525, Grenoble, France.

${ }^{2}$ CRIK3 (Centre de Recherche et d'Investigation en Kinésiologie, Kinésiopathologie et Kinésithérapie), Ecole de kinésithérapîe du CHU de Grenoble, France

${ }^{3}$ Service de Rhumatologie du CHU de Grenoble, France

* Auteur correspondant :

Ecole de kinésithérapie du CHU de Grenoble

BP 158

F 38130 Echirolles Cedex 
18 Key words: Manipulation; Foot; Ankle; Balance; Elderly.

\section{Abstract} effect induced by the suppression of vision.

The purpose of the present experiment was to investigate the effect of a therapeutic manipulation of the feet and ankles on postural control during quiet standing in elderly adults. Seventeen elderly adults stood barefoot on a force platform and were asked to sway as little as possible. Within a trial, vision was suppressed by eyes closure. The task was executed in two experimental sessions: before and after a therapeutic manipulation of the foot and ankle. Centre of foot pressure (COP) displacements along the mediolateral (ML) and anteroposterior (AP) axes were recorded. For the two experimental sessions, subjects exhibited comparable COP displacements when vision was available and were similarly affected by the suppression of vision. However, when subjects had to adapt to the absence of vision within a $10 \mathrm{~s}$ temporal frame, postural behavior became different in the two experimental sessions: The sum of the ML and AP COP displacements increased within the $10 \mathrm{~s}$ temporal frame before the therapeutic manipulation of the feet and ankles, whereas it remained unchanged after it. These results suggested that the therapeutic manipulation of the foot and ankle allows the elderly adults to partially compensate for the destabilising 


\section{Introduction}

Postural control is a particularly complex system involving various sensory and motor components. Among the sensory inputs relevant to the regulation of postural sway, the importance of somatosensory information from the feet and ankles is now well established (Dietz, 1992; Fitzpatrick et al., 1994; Meyer et al., 2004a, 2004b). Data supporting this view were obtained through experimental and clinical approaches. Experimental manipulations of somatosensory information from cutaneous, muscles, tendons and joints receptors were shown to have an effect on postural control, as evidenced either by cooling (Magnusson et al., 1990), anaesthetising (Thoumie \& Do, 1996; Meyer et al., 2004a, 2004b) or stimulating (Kavounoudias et al., 1998, 2001; Maurer et al., 2001) the plantar soles, by vibrating ankle muscles (Kavounoudias et al., 1998; Hay et al., 1996), by applying an ischemic block above the ankles (Fitzpatrick et al., 1994; Dierner et al., 1984), or by changing the characteristics of the supporting surface on which individuals are standing (Redfern et al., 1997; Robbins et al., 1998). Clinically, alteration or loss of somatosensory information from the lower limbs resulting from disease (diabetic peripheral neuropathy) is known to impair postural control (Simmons et al., 1997). Progressive degeneration of sensory inputs from the lower extremities also represents a common clinical finding associated with aging (Kenshalo, 1986; Skinner et al., 1984) and has even been identified as an important contributing factor to the occurrence of falls in elderly (Lord et al., 1991; Tinetti \& Speechley, 1989). Therefore, it is legitimate to propose that a therapeutic intervention designed to increase somatosensory function of the feet and ankles could be of great interest for controlling balance and preventing falls in the elderly. While the benefits of proprioceptive exercises are documented (Gauchard et al., 1999; Sinaki \& 
1 Lynn, 2002), it is surprising that there is an absence of data investigating whether and how

2 a therapeutic manipulation of the feet and ankles can modify postural control in elderly

3 adults.

The purpose of the present experiment was to improve the understanding of the contribution of distal lower limb inputs to the control of balance during functional activities. More precisely, it was to investigate the effects of a therapeutic manipulation of the feet and ankles on postural control during quiet standing in elderly adults. Since visual information is known to play an important role in postural control, particularly in elderly (Lord \& Menz, 2000; Sundermier et al., 1996), subjects' ability to regulate their postural sway was evaluated while being exposed to an abrupt suppression of visual information, as previously done by others (Hay et al., 1996; Teasdale et al., 1991). It was hypothesised that an "over-activation" of somatosensory information induced by a therapeutic manipulation of the feet and ankles allows the elderly persons to compensate for the destabilising effect of visual suppression. Since the somatosensory system includes various receptors that provide information about pressure distribution (cutaneous), muscle tension (Golgi tendon organs), joint angle changes (joint receptors) and muscle length changes (spindles), the intervention involved manual massage of the feet and mobilisation of both the feet and ankles joints.

\section{Methods}

17 male elderly adults (mean age $=74.5 \pm 9, .6$ years; mean body weight $=73.2 \pm$ $12.3 \mathrm{~kg}$; mean height $=165.6 \pm 9,3 \mathrm{~cm})$ participated in the experiment. Subjects were volunteers and gave written informed consent to the experimental procedure as required by the Helsinki declaration (1964) and the local Ethics Committee. None of the subjects 
1 presented any musculoskeletal problems, defects in the peripheral sensory system of the

2 lower extremities, vascular pathology, neurological disorders or vestibular impairment.

3 Finally, all subjects had normal or corrected-to-normal vision.

Subjects stood barefoot on a force platform, feet joined, and were asked to sway as little as possible, with their eyes open. Fifteen seconds into a trial, an auditory stimulus

6 directed the subjects to close their eyes. A trial finished with $10 \mathrm{~s}$ of no-vision. When vision

7 was available, subjects were asked to fixate the intersection of a black cross placed onto the

8 white wall distant $1 \mathrm{~m}$ in front of them, at the eyes level. When vision was not available,

9 they were asked to keep their gaze in a straight-ahead direction. This task was executed in

10 two experimental sessions (Pre- and Post test). Six $25 \mathrm{~s}$ trials were executed for each

11 experimental session, for a total of 12 trials. The duration of an experimental session lasted

$126 \mathrm{~min}$, approximately. The Pre-test served as a control session. In the Post-test session, the

13 measurements were performed immediately after a therapeutic manipulation of the feet and

14 ankles. This intervention, widely used by manual therapists (Dufour, 1996), was designed

15 to target the somatosensory system of the feet and ankles, using manual massage of the feet 16 and mobilisation of both the feet and ankles joints.

17 The aim of the massage was to enhance local blood circulation and to stimulate

18 cutaneous receptors. Massage technique application involved frictions and glide force

19 pressure of the plantar sole. Mobilisations elicited dorsiflexion and plantar flexion of the

20 mortise joints, eversion and inversion of the subtalar joints, anteroposterior glide, torsion,

21 flexion and extension of the midtarsal joints, anteroposterior glide and rotation of

22 tarsometatarsal joints, anteroposterior glide of intermetatarsal joints, plantar flexion and

23 extension of metarsophalangeal and interphalangeal joints. Manual massage and 
1 articulation techniques were applied to both the feet and ankles for $20 \mathrm{~min}$. Six $25 \mathrm{~s}$ trials were executed for each experimental session, for a total of 12 trials. The duration of an experimental session lasted 6 min, approximately. centre of foot pressure (COP). Signals from the force platform were sampled at $200 \mathrm{~Hz}$ (12

bit A/D conversion) and filtered with a second-order low-pass Butterworth filter $(10 \mathrm{~Hz}$ cut-off frequency).

CoP displacements (in millimetres) along the mediolateral (ML) and anteroposterior (AP) axes were used to estimate a global postural performance before and after the therapeutic manipulation. This measure corresponds to the sum of the displacement scalars over the sampling period. For convenience purposes, these two dependent variables will be referred to as ML and AP COP displacements, respectively. The first $10 \mathrm{sec}$ of a trial were used to allow subjects' stabilisation. COP displacements were analysed over three periods of $4 \mathrm{~s}$, immediately before the suppression of vision (T1), $1 \mathrm{~s}$ after the visual transition to prevent from the presumed effect of the visual transition from vision to no-vision (T2) and at the end of the trial (T3). Comparison of these three temporal frames yielded information on (1) the immediate effect of the suppression of vision on COP displacements (T1 vs. T2) and (2) the postural adaptation over time to the absence of vision (T2 vs. T3).

Analyses of variance (ANOVAs) were used for statistical comparison of the different conditions. Level of significance was set at 0.05 . Post-hoc analyses (NewmanKeuls) were used whenever necessary. 

21 data.

\section{Results}

Figure 1 illustrates representative COP displacements across a trial from a typical subject for each of the two Pre (left panel) and Post-test (left panel) experimental sessions.

\section{Postural control following the suppression of vision}

Insert Figure 1 about here

A preliminary analysis consisted in controlling whether the immediate effect of the suppression of vision on COP displacements was similar across the trials performed in the Pre-test session for the temporal frames T1 versus T2. The first three and the last three trials of the Pre-test session were compared using 2 Series (First 3 vs. Last 3 trials) $\times 2$ Temporal frames (T1 vs. T2) ANOVAs. For both the ML and AP COP displacements, results showed no effect of Series and no interaction of Series $\times$ Temporal frame $(P S>$ 0.05). This suggested that the immediate effect of the suppression of vision on COP displacements was constant across the Pre-test trials. Thus, data obtained in the Post-test session were not likely to be confounded with a possible effect of training or fatigue.

The purpose of the following analysis was to investigate whether subjects' ability to immediately adapt to the transition from vision to no vision was modified by the therapeutic manipulation of the feet and ankles. 2 Sessions (Pre vs. Post-test) $\times 2$ Temporal frames (T1 vs. T2) ANOVAs with repeated measures on both factors were applied to the 

21 least.

Analysis of the ML COP displacements showed a main effect of Temporal frame, with smaller ML COP displacements before than immediately after the suppression of vision $(\mathrm{F}(1,16)=27.58, P<0.001)$. The absence of an interaction of Session $\times$ Temporal frame $(P>0.05)$ showed that the therapeutic intervention did not affect ML COP displacements immediately after the visual transition (Figure 2A).

Insert Figure 2 about here

$(\mathrm{F}(1,16)=31.26, P<0.001)$ and showed a main effect of Session, yielding smaller AP

COP displacements in the Post- than in the Pre-test session $(\mathrm{F}(1,16)=8.01, P<0.05)$. The absence of an interaction of Session $\times$ Temporal frame $(P>0.05)$ also showed that the therapeutic intervention did not affect AP COP displacements immediately after the suppression of vision (Figure 2B).

To further investigate the persistence of the effect of the manipulation of the feet and ankles, the first three and the last three trials of the Post-test session were compared using 2 Series (First 3 vs. Last 3 trials) $\times 2$ Temporal frames (T1 vs. T2) ANOVAs. For both the ML and AP COP displacements, results showed no effect of Series and no interaction of Series $\times$ Temporal frame $(P s>0.05)$. This suggested that the therapeutic intervention on COP displacements was constant across the Post-test trials, for 6 min at 
A preliminary analysis controlled whether the postural adaptation to the suppression of vision within a $10 \mathrm{~s}$ temporal frame was similar in the trials performed in the Pre-test session for the temporal frames T2 versus T3. The first three and the last three trials of the Pre-test session were compared using 2 Series (First 3 vs. Last 3 trials) $\times 2$ Temporal frames (T2 vs. T3) ANOVAs. For both the ML and AP COP displacements, results showed no effect of Series $(P s>0.05)$, and no interaction of Series $\times$ Temporal frame $(P s>0.05)$. This suggested that postural adaptation to the suppression of vision within a $10 \mathrm{~s}$ temporal frame was constant across the Pre-test trials. This further confirmed that data obtained following the therapeutic manipulation were not likely to be confounded with a possible effect of training or fatigue.

The purpose of the following analysis was to investigate whether subjects' adaptation to the effect of the visual transition within a $10 \mathrm{~s}$ temporal frame was modified by the therapeutic intervention. Two Sessions (Pre vs. Post-test) $\times 2$ Temporal frames $(T 2$ vs. T3) ANOVAs with repeated measures on both factors were applied to the data.

Analysis of the ML COP displacements showed a significant interaction of Session $\times$ Temporal frame $(\mathrm{F}(1,16)=6.53, P<0.05)$. The decomposition of the interaction into its simple main effects showed that ML COP displacements increased over time in the Pre-test session, with smaller values immediately after the suppression of vision than after $6 \mathrm{~s}$ of adaptation in No-vision $(P<0.05)$, whereas they remained unchanged in the Post-test session $(P>0.05)$ (Figure 2A).

Analysis of the AP COP displacements showed a significant interaction of Session $\times$ Temporal frame $(\mathrm{F}(1,16)=5.48, P<0.05)$. The ANOVA also confirmed main effects of 
1 Temporal frame $(\mathrm{F}(1,16)=4.53, P<0.05)$ and Session $(\mathrm{F}(1,16)=11.07, P<0.01)$. The

2 decomposition of the interaction into its simple main effects showed that AP COP

3 displacements increased over time in the Pre-test session, with smaller values immediately

4 after the suppression of vision than after $6 \mathrm{~s}$ of adaptation in No-vision $(P<0.01)$, whereas

5 they remained unchanged in the Post-test session $(P>0.05)$ (Figure 2B).

6 To further investigate the persistence of the effect of the therapeutic manipulation of

7 the feet and ankles, the first three and the last three trials of the Post-test session were

8 compared using 2 Series (First 3 vs. Last 3 ) $\times 2$ Temporal frames (T2 vs. T3) ANOVAs.

9 For both ML and AP COP displacements, results showed no effect of Series $(P s>0.05)$,

10 and no interaction of Series $\times$ Temporal frame $(P s>0.05)$. This suggested that the benefits

11 of the therapeutic intervention on COP displacements was constant across the Post-test

12 trials, for 6 min at least.

13 Discussion

14 The purpose of the present experiment was to investigate the effects of a therapeutic 15 manipulation of the feet and ankles on postural control during quiet standing in elderly

16 adults. Subjects' ability to maintain a stable upright posture was evaluated while facing

17 changes in the availability of visual information before and after the therapeutic

18 intervention. Withdrawing visual information forced the subjects to reorganise the

19 hierarchy of sensory information since vestibular and proprioceptive inputs became the

20 only remaining sources of sensory information available for regulating posture.

21 Before and after the therapeutic manipulation, subjects exhibited comparable COP

22 displacements when vision was available (temporal frame T1), suggesting that the massage 
1 and mobilisation of the feet and ankles had no immediate effect on postural stability and/or that vision largely compensated for the presumed benefits of a therapeutic manipulation.

3 Previous studies showed similar compensations following fatigue of the lower limbs

4 (Vuillerme et al., 2001). Similarly, the therapeutic manipulation did not affect COP displacements immediately following the visual transition from vision to no-vision

6 (temporal frame T2). This absence of effect may be due to the limited impact of the therapeutic manipulation with respect to the amplitude of normal body oscillations in the absence of vision.

Interestingly, it is when adapting to this absence of vision within a $10 \mathrm{~s}$ temporal frame that the postural behaviour in the two experimental sessions became different

11 (temporal frame T3). After $10 \mathrm{~s}$ of adaptation, postural instability still increased in the control session whereas following the therapeutic manipulation subjects seemed able to

13 provide a rapid recalibration and reorganisation of postural control to take into account the new sensory conditions by switching from visual to proprioceptive control. This adaptive mechanism would ensure a stabilisation of subjects' postural behaviour following the withdrawal of vision.

With regard to the hypothesis of a global "over-activation" of somatosensory

18 information induced by the therapeutic manipulation of the feet and ankles, the present

19 results are in line with those of Nougier et al. (1997) when altering somatosensory inputs

20 by means of a foam support surface. In their experiment, postural control in young adults

21 was analysed in different conditions of vision and two conditions of ankle proprioceptive

22 information (normal and altered support surface with a $5 \mathrm{~cm}$ thick foam support). When

23 somatosensory information was not altered, as in the Post-test session, young subjects 
1 adapted to the absence of vision. Conversely, when the proprioceptive inputs were altered

2 by the use of the foam support surface, as in the Pre-test session, subjects showed no

3 adaptation. Altogether, these results and the present ones demonstrate that the quality of

4 somatosensory information plays a major role in compensating for the absence of vision. In

5 the present case, the therapeutic manipulation seemed to partially compensate for the

6 observed alteration of somatosensory information with age (Kenshalo, 1986; Skinner et al.,

7 1984), by temporarily increasing subjects' sensitivity to these inputs.

8 In conclusion, the present findings showed that elderly adults can benefit from a

9 therapeutic manipulation of the feet and ankles, even though estimated for a short time, for

10 regulating their postural sway during quiet standing. This suggested that such

11 manipulations may allow people to overcome or compensate for functional difficulties due

12 to age and more generally to disease-related sensory loss. It remains, however, to further

13 investigate the underlying neurophysiological mechanisms and to better locate the sites of

14 these effects . It remains also to further investigate the overall duration of the observed

15 benefits in order to validate the utility of these various manipulations and to better specify

16 the most efficient characteristics of a training/rehabilitation program that would include this

17 kind of manipulation. 

$18 \quad$ (1986) 632-642.

19

\section{References} 46 (2000) 306-310.

H.C. Diener, B. Dichgans, B. Guschlbauer, H. Mau, The significance of proprioception on postural stabilization as assessed by ischemia. Brain Res. 296 (1984) 103-109.

V. Dietz, Human neuronal control of automatic functional movements: interaction between central programs and afferent input. Physiol. Rev. 72 (1992) 33-69.

M. Dufour, Massages, Encycl. Méd. Chir. Paris, Elsevier 1996.

R. Fitzpatrick, D.K. Rogers, D.I. McCloskey, Stable human standing with lower-limb muscle afferents providing the only sensory input. J. Physiol. 480 (1994) 395-403.

G. Gauchard, C. Jeandel, A. Tessier, P. Perrin, Beneficial effect of proprioceptive physical activities on balance control in elderly human subjects. Neurosci. Lett. 273 (1999) 81-84.

L. Hay, C. Bard, M. Fleury, N. Teasdale, Availability of visual and proprioceptive afferent messages and postural control in elderly adults. Exp. Brain Res. 108 (1996) 129-139.

A. Kavounoudias, R. Roll, J.P. Roll, Foot sole and ankle muscle inputs contribute jointly to human erect posture regulation. J. Physiol. 532 (2001) 869-878.

A. Kavounoudias, R. Roll, J.P. Roll, The plantar sole is a "dynamometric map" for human balance control. Neuroreport 9 (1998) 3247-3252.

D.R. Kenshalo, Somesthetic sensitivity in young and elderly humans. J. Gerontol. 41

S.R. Lord, R.D. Clark, I.W. Webster, Physiological factors associated with falls in an elderly population. J. Am. Geriatr. Soc. 39 (1991) 1194-1200.

1 S.R. Lord, H.B. Menz, Visual contributions to postural stability in older adults. Gerontol. 
1 M. Magnusson, H. Enbom, R. Johansson, I. Pyykko, Significance of pressor input from the human feet in anterior-posterior postural control. The effect of hypothermia on vibration

3 induced body-sway. Acta Otolaryngol. Stockh. 110 (1990) 182-188.

4 C. Maurer, T. Mergner, B. Bolha, F. Hlavacka, Human balance control during cutaneous

$5 \quad$ stimulation of the plantar soles. Neurosci. Lett. 302 (2001) 45-48.

$6 \quad$ P.F. Meyer, L.I. Oddsson, C.J. De Luca, Reduced plantar sensitivity alters postural

7 responses to lateral perturbations of balance. Exp. Brain Res. 157 (2004) 526-36.

8 P.F. Meyer, L.I. Oddsson, C.J. De Luca, The role of plantar cutaneous sensation in

9 unperturbed stance. Exp. Brain Res. 156 (2004) 505-12.

10 V. Nougier, C. Bard, M. Fleury, N. Teasdale, Contribution of central and peripheral vision

11 to the regulation of stance. Gait and Posture 5 (1997) 34-41.

12 M.S. Redfern, P.L. Moore, C.M. Yarsky, The influence of flooring on standing balance 13 among older persons. Hum. Factors 39 (1997) 445-455.

14 S. Robbins, E. Waked, N. Krouglicof, Improving balance. J. Am. Geriatr. Soc. 46 (1998)

$15 \quad 1363-1370$.

16 R.W. Simmons, C. Richardson, R. Pozos, Postural stability of diabetic patients with and

17 without cutaneous sensory deficit in the foot. Diabetes Res. Clin. Pract. 36 (1997) 153-160.

18 M. Sinaki, S.G. Lynn, Reducing the risk of falls through proprioceptive dynamic posture

19 training in osteoporotic women with kyphotic posturing: a randomized pilot study. Am. J.

20 Phys. Med. Rehabil. 81 (2002) 241-246.

21 H.B. Skinner, R.L. Barrack, S.D. Cook, Age-related decline in proprioception. Clin.

22 Orthop. 184 (1984) 208-211. 
1 L. Sundermier, M.H. Woollacott, J.L. Jensen, S. Moore, Postural sensitivity to visual flow

2 in aging adults with and without balance problems. J. Gerontol. A Biol. Sci. Med. Sci. 51

3 (1996) M45-M52.

4 N., Teasdale G.E. Stelmach, A. Breunig, Postural sway characteristics of the elderly under

5 normal and altered visual and support surface conditions. J. Gerontol. 46 (1991) B338-

$6 \quad$ B244.

$7 \quad$ P. Thoumie, M.C. Do, Changes in motor activity and biomechanics during balance

8 recovery following cutaneous and muscular deafferentation. Exp. Brain Res .110 (1996)

$9 \quad 289-297$.

10 M.E. Tinetti, M. Speechley, Prevention of falls among the elderly. N. Engl. J. Med. 320

11 (1989) 1055-1059.

12 N. Vuillerme, V. Nougier, J.-M. Prieur, Can vision compensate for a lower limbs muscular 13 fatigue for controlling posture? Neurosci. Lett. 308 (2001) 130-106. 


\section{$1 \quad$ Figures captions}

2 Figure 1. Representative displacements of COP along the mediolateral (A) and

3 anteroposterior (B) axis for the two Pre- and Post-test sessions. The dotted lines indicate the

4 suppression of vision.

5

6

7 Figure 2. Mean COP displacements and standard deviation along the two mediolateral $(A)$

8 and anteroposterior $(B)$ axes and for the two Pre and Post-test sessions. The two

9 experimental sessions are presented with different symbols: Pre-test (white diamonds) and

10 Post-test (black squares). 


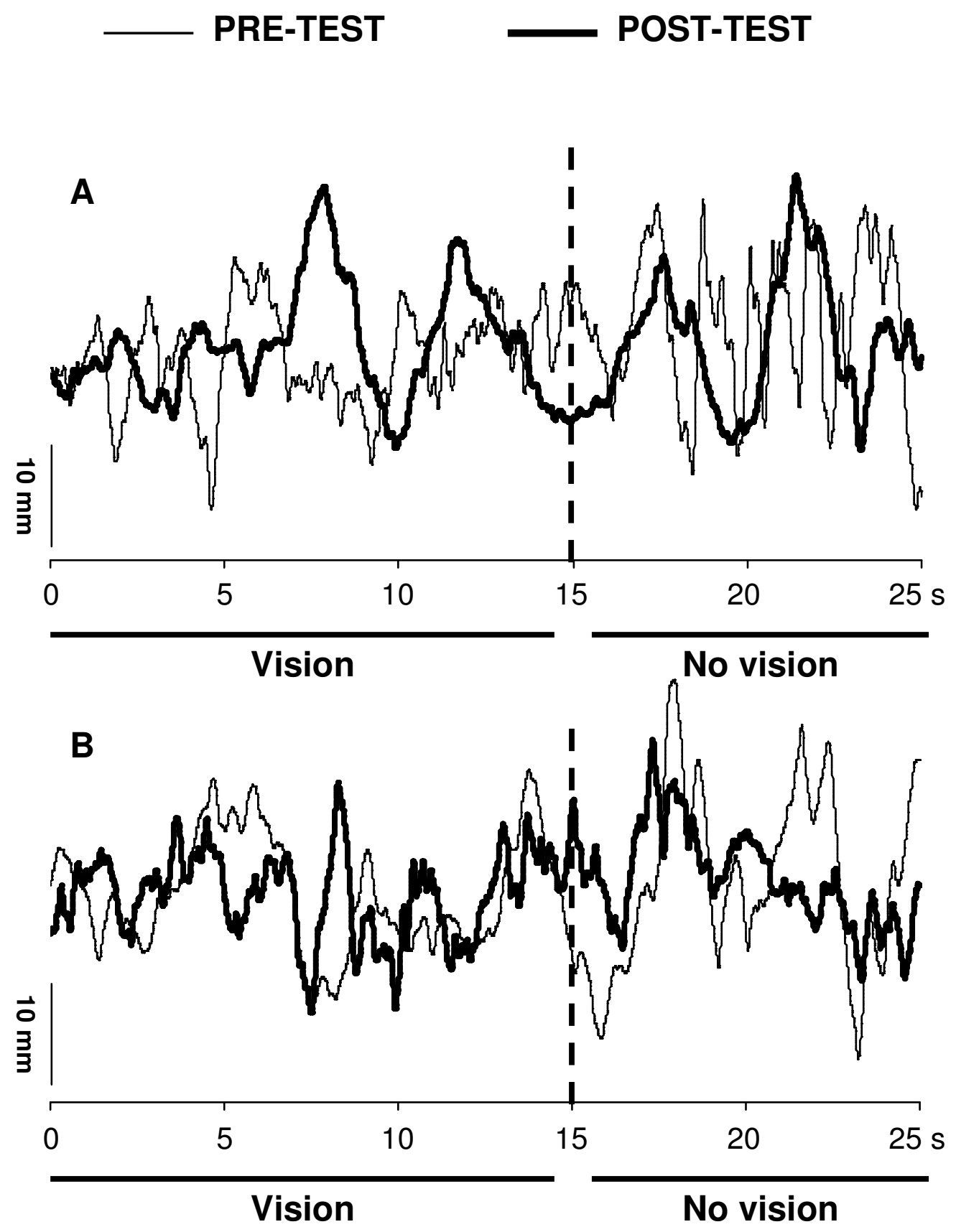




\section{$\checkmark$ PRE-TEST - - POST-TEST}
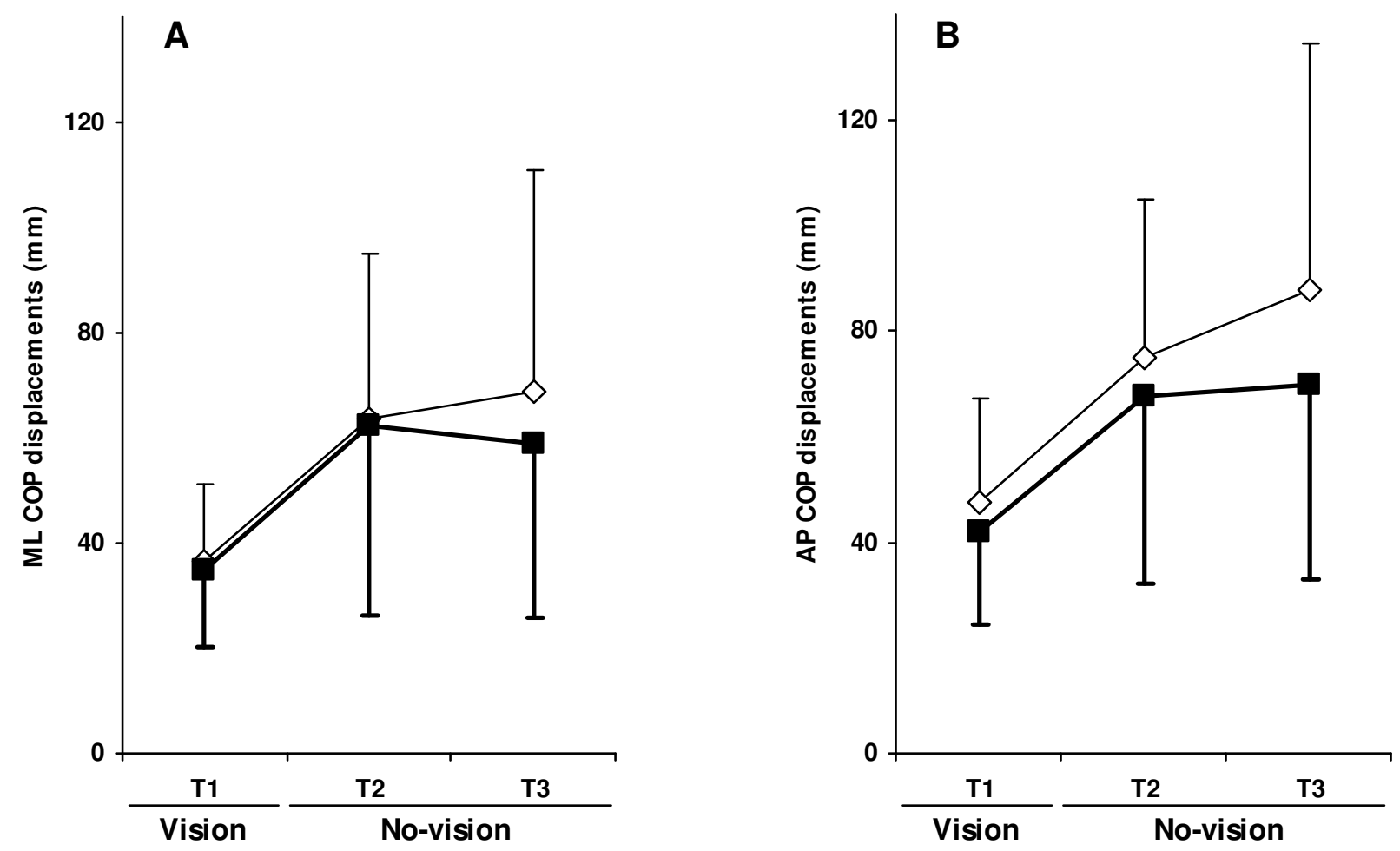